\title{
Tenascin-c knockdown suppresses vasculogenic mimicry of gastric cancer by inhibiting ERK- triggered EMT
}

\author{
Xing Kang ${ }^{1,4}$, En Xu $\mathbb{1}^{2,4}$, Xingzhou Wang ${ }^{2,4}$, Lulu Qian ${ }^{1,4}$, Zhi Yang ${ }^{1}$, Heng Yu', Chao Wang ${ }^{2}$, Chuanfu Ren ${ }^{1}$, Yizhou Wang ${ }^{1}$,

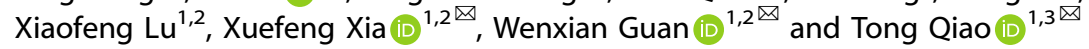

(c) The Author(s) 2021, corrected publication 2021

\begin{abstract}
Gastric cancer is one of the most common malignancies worldwide and vasculogenic mimicry (VM) is considered to be the leading cause for the failure of anti-angiogenesis therapy in advanced gastric cancer patients. In the present study, we investigate the role of tenascin-c (TNC) in the formation of VM in gastric cancer and found that TNC was upregulated in gastric cancer tissue than in the corresponding adjacent tissues and correlated with VM and poor prognosis of gastric cancer. Furthermore, knockdown of TNC significantly inhibited VM formation and proliferation of gastric cancer cells in vitro and in vivo, with a reduction in cell migration and invasion. Mechanistically, TNC knockdown suppressed the phosphorylation of ERK and subsequently inhibited the process of EMT, both of which play an important role in VM formation. Our results indicated that TNC plays an important role in VM formation in gastric cancer. Combining inhibition of TNC and ERK may be a potential therapeutic approach to inhibit gastric cancer growth and metastasis and decrease antiangiogenic therapeutic resistance.
\end{abstract}

Cell Death and Disease (2021) 12:890; https://doi.org/10.1038/s41419-021-04153-1

\section{INTRODUCTION}

Gastric cancer is a malignant tumor originating from the mucosal epithelium of stomach. In China, the incidence of gastric cancer ranks second and the mortality rate ranks third [1]. Approximately 1.2 million new cases of gastric cancer occur each year worldwide, and China accounts for about $40 \%[2,3]$. The proportion of early gastric cancer is low (about 40\%), and most of them are already advanced (about 60\%) when diagnosed, due to lack of early clinical symptoms [4]. The treatment options for advanced gastric cancer are few and the efficacy is limited and the 5-year survival rate is $<20 \%$ [5]. Even though the diagnosis and treatment of gastric cancer have improved these years, the prognosis of patients is still poor. Gastric cancer recurrence and metastasis remain the most common cause of poor long-term survival [6]. Tumor growth and metastasis require an adequate blood supply. However, antivascular therapy did not improve the survival in patients with advanced gastric cancer. In addition, antiangiogenic therapy has been hypothesized to induce the formation of vasculogenic mimicry (VM) [7], without the participation of endothelial cells in the formation of the blood supply pattern. Thus, VM may play an important role in the development of GC and may be a major cause of failure in antiangiogenic therapy.

VM was first discovered in melanoma in 1999 [8]. It is a vascular-like structure that can mimic the embryonic vascular network pattern to nourish the tumor tissue [9]. The structure formed by VM is composed of a basement membrane and lined by cancer cells without endothelial cells on the inner wall, allowing blood plasma and red blood cells to flow [10]. VM structure serves as an adjunct to the vasculature system, helping tumor to grow. Thus, due to direct exposure to the blood, cancer cells can easily leak out and migrate through the bloodstream to metastasize to other organs [11]. Recent studies have shown that VM is observed and associated with poor prognosis in many malignant tumors, including breast cancer [12], hepatocellular cancer [13], osteosarcoma [14], colorectal cancer [15] and gastric cancer [16, 17]. However, few studies have considered VM as a potential therapeutic target in gastric cancer, and the mechanisms underlying VM remain unknown.

Tenascin-C (TNC) is a hexameric, multimodular extracellular matrix protein and is the founding member of the tenascin gene family [18]. The synthesis of TNC is tightly regulated and the distribution of TNC is highly restricted in adult tissues. However, it is specifically upregulated during wound healing, chronic inflammation or cancer [19]. Recent studies have shown that the expression of TNC was significantly associated with pT stage, lymph node metastasis and distant metastasis in gastric cancer [20]. TNC was identified in inducing epithelial-tomesenchymal (EMT) transition [21] and EMT has been found to play a key role in VM formation $[9,22,23]$. However, the role of TNC in VM formation and EMT in gastric cancer was not reported.

\footnotetext{
'Department of General Surgery, Nanjing Drum Tower Hospital Clinical College of Nanjing Medical University, No. 321 Zhongshan Road, 210008 Nanjing, China. ${ }^{2}$ Department of General Surgery, Affiliated Drum Tower Hospital, Medical School of Nanjing University, No. 321 Zhongshan Road, 210008 Nanjing, China. ${ }^{3}$ Department of Vascular Surgery, The Affiliated Drum Tower Hospital of Nanjing University Medical School, No. 321 Zhongshan Road, 210008 Nanjing, China. ${ }^{4}$ These authors contributed equally: Xing Kang, En Xu,

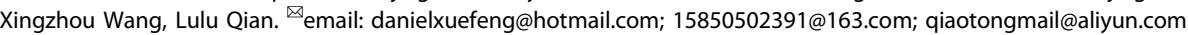
Edited by Professor Nickolai Barlev
}

Received: 18 February 2021 Revised: 16 August 2021 Accepted: 9 September 2021 Published online: 29 September 2021 
In this study, we therefore investigated the role of TNC in VM formation in gastric cancer cell lines. We used siRNA to knock down the expression of TNC and evaluated the changes in VM formation and EMT markers. Our findings suggest that TNC may represent a novel target in the treatment of gastric cancer.

\section{MATERIALS AND METHODS Clinical samples}

Fifty pairs of gastric cancer tissues and corresponding adjacent tissues and 66 gastric cancer samples from patients who underwent surgery more than 5 years were obtained from the General Surgery Department of the Nanjing Drum Tower Hospital. The research protocol complies with the ethical guidelines of the 1975 Declaration of Helsinki. All experiments with human samples have been reviewed and approved by the Ethics Committee and Animal Welfare Committee of Nanjing Drum Tower Hospital. Written informed consent was obtained from each participant before learning.

\section{Immunohistochemical (IHC) staining}

The tissue is fixed in formalin and embedded in paraffin, and then cut into thin slices. The sections were deparaffinized with xylene and hydrated with ethanol, and then the antigen was recovered by pressure cooking. At room temperature, the sections were sliced with TNC primary antibody (ab108930, Abcam, Cambridge, MA), MMP9 (Abcam, ab76003, 1:1000 dilution), MMP2 (Abcam, ab92536, 1:1000 dilution) and CD31 (ab134168, Abcam), incubated for $60 \mathrm{~min}$ and then incubated with IgG H\&L (HRP; 1:200 dilution, Abcam, Cambridge, UK). Then, the sections were stained with chromogen and counterstained with hematoxylin. The score is based on the intensity of staining $(0$ means no staining, 1 means weak staining, 2 means moderate staining, and 3 means strong staining). The product of the two levels was calculated as the final expression score.

\section{Cell culture}

Three human gastric cancer cell lines, AGS, BGC-823 and Hs746T, were purchased from the American Type Culture Collection (Manassas, VA, USA). The AGS, BGC-823 and Hs746T cells were supplemented with $10 \%$ fetal bovine serum (FBS; Gibco, Waltham, MA, USA) in RPMI 1640 medium (Gibco, Waltham, MA, USA) and 1\% penicillin/streptomycin (Gibco, Waltham, MA, USA). The medium was refreshed and the cells were passed every 3 days.

\section{siRNA transfection assay}

According to the manufacturer's instructions, GC cells were transfected with siRNA (Guangzhou Ribobio Co., Ltd.) specifically targeting TNC using interferon reagent (Polyplus, New York, USA). Firstly, 5 pmol of siRNA was diluted in $200 \mu \mathrm{L}$ of medium without serum, vortexed for $20 \mathrm{~s}$, and centrifuged briefly. Then, $8 \mu \mathrm{L}$ of interferin reagent was added, vortexed for $20 \mathrm{~s}$, centrifuged briefly, and then incubated for $10 \mathrm{~min}$ at room temperature. During the incubation time, $2 \mathrm{~mL}$ of serum-containing medium was prepared. Finally, the transfection mix was added to the cells in serum-containing medium. The siRNA target sequence is TAACCATTTCCGACATTAA. The knockdown efficiency of TNC siRNA was checked by western blot.

\section{Lentiviral transduction}

Control lentivirus vectors and lentiviral vector encoding a gene-specific shRNA against TNC's scrambled shRNA were purchased from Shanghai Gene-Pharma Co., Ltd (Shanghai, China). The human GC cell line was transduced with lentiviral particles along with polybrene, and screened with puromycin $(1 \mathrm{mg} / \mathrm{mL}$ ) (Thermo Fisher Scientific, Waltham, MA, USA) for 2 weeks. The knockdown efficiency was detected by western blot.

\section{Western blot analysis}

The cell lysates were collected, and $30 \mathrm{mg}$ protein of each sample was separated using $10 \%$ SDS-PAGE. The protein was then electrotransferred onto Polyvinylidene Fluoride (PVDF) membrane (Millipore, Boston, MA, USA). The PVDF membrane was sealed in 10\% skimmed milk. Then the membrane was incubated with primary antibodies,
Table 1. Primer used in quantitative real-time PCR assays.

\begin{tabular}{|ll}
\hline Primer & Sequences $\left(\mathbf{5}^{\prime} \rightarrow \mathbf{3}^{\prime}\right)$ \\
\hline GAPDH-F & GGAGTCCACTGGCGTCTTCA \\
\hline GAPDH-R & GTCATGAGTCCTCCACGATACC \\
\hline TNC-F & TCCCAGTGTTCGGTGGATCT \\
\hline TNC-R & TTGATGCGATGTGTGAAGACA \\
\hline E-cadherin-F & CGAGAGCTACACGTTCACGG \\
\hline E-cadherin-R & GGGTGTCGAGGGAAAAATAGG \\
\hline N-cadherin-F & AGCCAACCTTAACTGAGGAGT \\
\hline N-cadherin-R & GGCAAGTTGATTGGAGGGATG \\
\hline Vimentin-F & GACGCCATCAACACCGAGTT \\
\hline Vimentin-R & CTTTGTCGTTGGTTAGCTGGT \\
\hline Slug-F & TGTGACAAGGAATATGTGAGCC \\
\hline Slug-R & TGAGCCCTCAGATTTGACCTG \\
\hline Snail-F & TCGGAAGCCTAACTACAGCGA \\
\hline Snail-R & AGATGAGCATTGGCAGCGAG \\
\hline Twist1-F & GTCCGCAGTCTTACGAGGAG \\
\hline Twist1-R & GCTTGAGGGTCTGAATCTTGCT \\
\hline MMP9-F & GGGACGCAGACATCGTCATC \\
\hline MMP9-R & TCGTCATCGTCGAAATGGGC \\
\hline MMP2-F & GATACCCCTTTGACGGTAAGGA \\
\hline MMP2-R & CCTTCTCCCAAGGTCCATAGC \\
\hline
\end{tabular}

including anti-TNC (Abcam, ab108930, 1:1000 dilution), anti-GAPDH (Abcam, ab181602, 1:10,000 dilution), and anti-ERK (Abcam, ab184699, 1:1000 dilution)), anti-p-ERK (Abcam, ab201015, 1:1000 dilution), antiMMP9 (Abcam, ab76003, 1:1000 dilution), anti-MMP2 (Abcam, ab92536, 1:1000 dilution), anti-E-cadherin (Abcam, ab1416, 1:1000 dilution), anti$\mathrm{N}$-cadherin (Abcam, ab18203, 1:1000 dilution), anti-vimentin (Abcam, ab92547, 1:1000 dilution), anti-snail (Abcam, ab216347, 1:1000 dilution), anti-clumping (Abcam, ab27568, 1:1000 dilution) and anti-TWIST1 (Abcam, ab50887, 1:1000 dilution) overnight. Then the membrane and horseradish peroxidase-conjugated secondary antibody (Abcam $\mathrm{AB6721}, 1: 10,000$ dilution) were incubated for $2 \mathrm{~h}$ at room temperature. Chemiluminescence detection reagent (Millipore, Boston, MA, USA) was used to visualize protein bands.

\section{Reverse transcription quantitative real-time polymerase chain reaction (RT-qPCR)}

Total RNA was extracted from GC cells, and then reverse transcribed into CDNA using an RT-PCR kit (Vazyme, China). Gene-specific primers were designed using Primer Express version 2.0 software (Applied Biosystems Inc., Milan, Italy), and are listed in Table 1. RT-PCR analysis was performed using SYBR Green Premix Ex Taq. Human GAPDH was used as an internal reference gene. The relative mRNA expression was calculated according to the $2^{-\Delta \Delta \mathrm{Ct}}$ method.

\section{Bioinformatics analysis}

We used the ACRG database to analyze the relationship between TNC expression and the prognosis of gastric cancer. The gene expression data were derived from The Cancer Genome Atlas (TCGA; https://portal.gdc. cancer.gov/projects/TCGA-LIHC) and analyzed using Gene Set Enrichment Analysis (GSEA).

\section{Cell viability assays}

The AGS, BGC-823 and Hs746T cell lines were seeded into 96-well microplates at a density of 5000 cells per well. The cell proliferation was determined using the Cell Counting Kit 8 (CCK-8) assay (Dojindo, Kumamoto, Japan) according to the manufacturer's instructions. Briefly, $10 \mu \mathrm{L}$ of LCCK-8 working solution was added per $100 \mu \mathrm{L}$ of medium to the microtiter plate and the cells were incubated for $1.5 \mathrm{~h}$. The OD450 value was determined by using the MRX II microplate reader (Dynex, Chantilly, VA, USA). 


\section{EdU staining}

The GC cells were incubated with $10 \mu \mathrm{M}$ Edu (C0071L, Beyotime, Shanghai, PR China) for $2 \mathrm{~h}$, and fixed with $4 \%$ paraformaldehyde for $15 \mathrm{~min}$ at room temperature. Then, the GC cells were washed with $3 \%$ Bovine serum albumin (BSA) in Phosphate Buffered Saline (PBS) $3 \times 5$ min, and containing $0.3 \%$ Triton PBS X-100 penetration for $10-15 \mathrm{~min}$. The cells were washed thoroughly with PBS containing 3\% BSA, and then incubated with Click Additive Solution for $30 \mathrm{~min}$ in the dark. Next, the cells were washed with PBS containing 3\% BSA for $3 \times 5$ min, and then incubated with Hoechst 33342 for $10 \mathrm{~min}$ in the dark. Finally, the cells were washed and observed under an Olympus microscope.

\section{Cell cycle analysis}

GC cells were treated with propidium iodide ( $\mathrm{Pl}$; Dawen) and were analyzed by flow cytometry. Modfit Software (Verity Software House) was used for quantitative analysis of the cell cycle.

\section{Cell apoptosis analysis}

The death of apoptotic cells was detected by flow cytometry using Annexin V-FITC Apoptosis Detection Kit II (KeyGEN BioTECH, Nanjing, China). The cells were harvested and washed twice with PBS. Then, cells were resuspended with $5 \mu \mathrm{L}$ Annexin V-FITC and $500 \mu \mathrm{L} 1 \times 5 \mu \mathrm{L} \mathrm{PI}$ binding buffer, and incubated for $15 \mathrm{~min}$ in the dark at room temperature. Then, the percentage of apoptotic cells was analyzed by flow cytometry.

\section{Wound-healing assay}

The cells were seeded in a six-well plate at a density of $5 \times 10^{5} /$ well and cultured to $90 \%$ confluence. A plastic spatula was used to make a wound track on each plate, wash the plate with PBS to remove loose cell debris and replenish the plate with fresh low-serum culture medium (1\% FBS). After $48 \mathrm{~h}$ of incubation, an Olympus microscope was used to measure the migration distance and calculate the migration rate. All experiments were repeated three times.

\section{Cell migration assay}

The Transwell chamber (Corning) was used to evaluate migration in Transwell analysis. The cells were seeded in the upper cavity at a density of $5 \times 10^{4} /$ cavity, and RPMI- 1640 containing $10 \%$ fetal calf serum was added as a chemotactic agent in the lower cavity. After $24 \mathrm{~h}$ of incubation at $37^{\circ} \mathrm{C}$, the cells in the upper chamber were removed with cotton swabs, and the cells in the lower chamber were fixed with formaldehyde for $30 \mathrm{~min}$ and stained with $0.1 \%$ crystal violet for $20 \mathrm{~min}$. All experiments were repeated three times. Ten fields of view were randomly selected, and the positively stained cells were counted using an Olympus microscope.

\section{Cell invasion assay}

The Transwell chamber was precoated ( $6.5 \mathrm{~mm}$, Costar, Corning, NY, USA) with Matrigel for $30 \mathrm{~min}$. Then, $1 \times 10^{5}$ cells suspended in serum-free RPMI 1640 medium were inoculated into the upper chamber, and RPMI 1640 medium containing $10 \%$ FBS was added to the lower chamber. After incubating for $24 \mathrm{~h}$ at $37^{\circ} \mathrm{C}$, the cells in the upper chamber were removed with a cotton swab, the cells in the lower chamber were fixed with formaldehyde for $30 \mathrm{~min}$, and stained with $0.1 \%$ crystal violet for $20 \mathrm{~min}$. All experiments were repeated three times. Ten fields of view were randomly selected, and the positively stained cells were counted using an Olympus microscope.

\section{Three-dimensional culturing}

Three-dimensional culture was used to evaluate the formation of VM. Fifty microliters of Matrigel (BD Biosciences, Sparks, MD, USA) was added to a 96 -well plate and incubated at $37^{\circ} \mathrm{C}$ for $30 \mathrm{~min}$. Then, the cell suspension was coated on Matrigel and incubated at $37^{\circ} \mathrm{C}$ for $8 \mathrm{~h}$. A brightfield microscope was used to image five random fields of view of each well.

\section{Pseudopodia formation detection}

The GC cells were seeded on glass slides for $24 \mathrm{~h}$ and cultured. The cells were then fixed with $4 \%$ paraformaldehyde and infiltrated with $0.3 \%$ Triton $\mathrm{X}-100$. F-actin is an important pseudopodia component and is labeled with phalloidin labeled with Alexa Fluor 555 (A34055, Invitrogen, USA). Then the instructions were followed for Cortactin and DAPI (4,6-dimidyl-2-phenylindole) labeling. The slides were fixed with ProLongTM Gold Antifade Mountant (P36930 from Life Technologies, USA). FV3000 confocal laser scanning microscope (Olympus Japan) was used to obtain confocal micrographs under the control of supporting software.

\section{Xenograft and peritoneal dissemination model}

Four-week-old female BALB/c nude mice were purchased from the Model Animal Research Center of Nanjing University and placed in a pathogenfree environment in the animal laboratory of Nanjing University. For the xenograft model, TNC knockdown and control GC cells $\left(2 \times 10^{6}\right)$ were resuspended in $200 \mu \mathrm{L}$ PBS. Then injected into the ventral side of nude mice (5 mice/group). Twenty-one days after tumor cell implantation, mice were sacrificed. The tumor was removed and weighed at autopsy. For the peritoneal diffusion model, $400 \mu \mathrm{L}$ of TNC knockdown and control GC cells $\left(3 \times 10^{6}\right)$ in PBS were injected into the peritoneal cavity. The peritoneal metastasis was checked and recorded when the mice were sacrificed on the 14th day after injection. No randomization was used and no blinding was done in this experiment.

\section{Statistical analysis}

We used SPSS 22.0 (IBM) to statistically analyze the data. Three or more independent experiments are expressed as mean \pm standard deviation. The comparison between the two groups was performed using the Student's $t$ test. One-way analysis of variance was used to compare the three groups. Counting data were compared by chi-square test. Linear regression was used to analyze the correlation between the two variables. The difference was considered statistically significant at a $P$ value of $<0.05$

\section{RESULTS}

TNC was upregulated in gastric cancer tissues and correlated with poor prognosis in gastric cancer patients

To evaluate TNC expression in gastric cancer tissues, we used immunohistochemistry to examine TNC expression in 50 pairs of gastric cancer tissues and corresponding adjacent tissues. TNC was mainly distributed in cytoplasm and extracellular matrix (Fig. $1 \mathrm{~A})$ and was significantly upregulated in cancer tissues than in normal tissues (Fig. 1B). Furthermore, we used western blot to examine the protein level of TNC in eight randomly selected gastric cancer tissues paired with adjacent tissues and demonstrated that TNC was significantly upregulated in gastric cancer tissues (Fig. 1C). Then, we used IHC to evaluate the TNC, PAS and CD31 expression in gastric cancer tissues. The results showed that VM exists in gastric cancer, consistent with previous studies $[24,25]$, and TNC expression had a positive correlation with VM in gastric cancer tissues (Fig. 1D). In addition, analysis of the association between TNC and prognosis of gastric cancer based on ACRG cohort revealed that patients with higher TNC levels had significantly shorter overall survival (OS) and relapse-free survival (RFS) compared to those with low TNC expression (Fig. 1E, F). Then we evaluated the expression of TNC in 66 postoperative specimens of gastric cancer patients and found that TNC expression was significantly correlated with tumor size, AJCC stage, $\mathrm{T}$ stage and $\mathrm{N}$ stage (Table 2). What's more, Kaplan-Meier analysis also indicated that patients with high TNC expression had a shorter survival (Fig. 1G). These results indicated that the expression of TNC is correlated with cancer grade and may predict poor prognosis of gastric cancer patients.

\section{Construction of cell lines and TNC knockdown attenuated VM formation in vitro}

We examined the expression of TNC by western blot in five gastric cancer cell lines, including MKN-45, AGS, Hs746T, BGC-823 and MGC- 803 and found that TNC was highly expressed in AGS, BGC-823 and Hs746T cell lines (Fig. 2A). Thus, AGS, BGC-823 and Hs746T cells were selected to evaluate the efficiency of siRNA- 
A

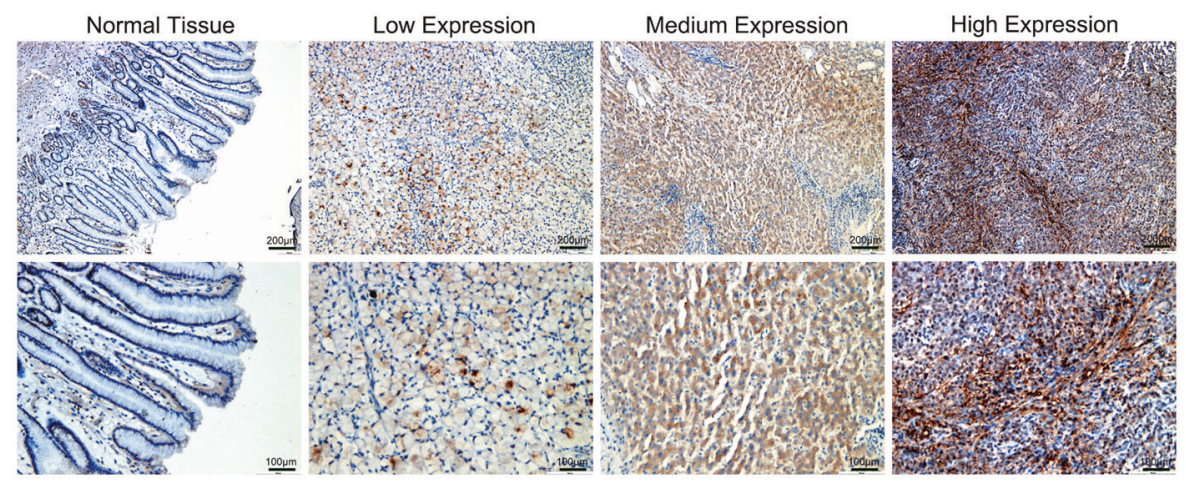

B
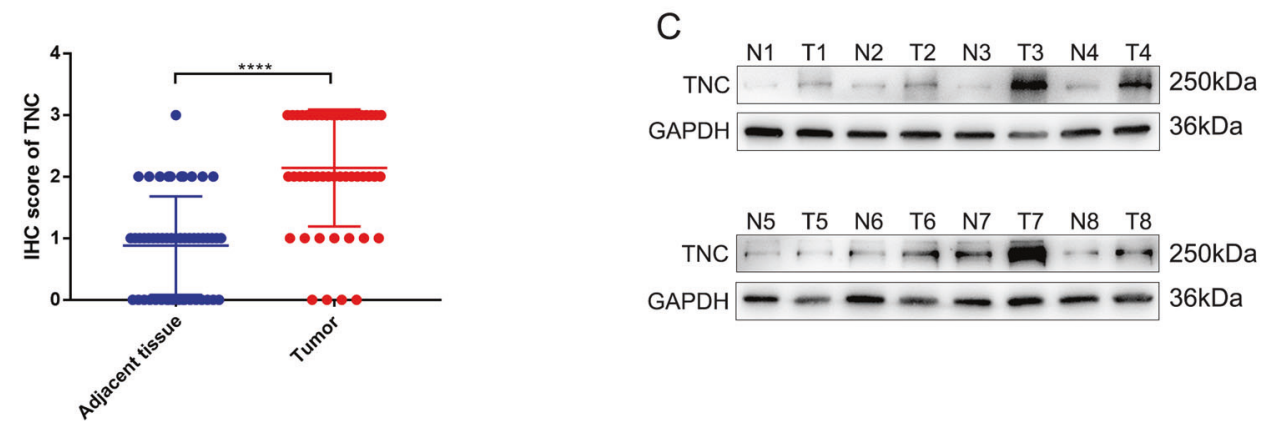

$\mathrm{D}$

TNC

\section{CD31/PAS}
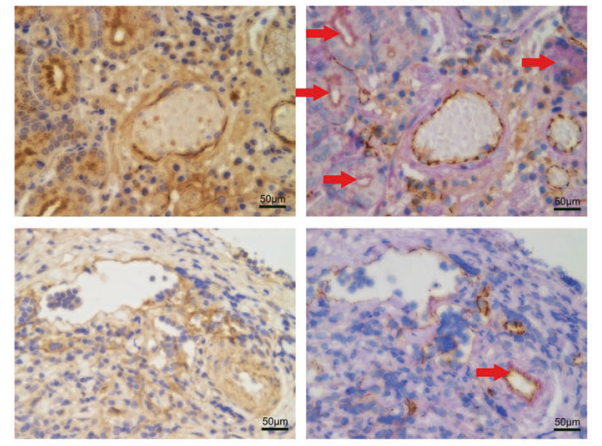

E

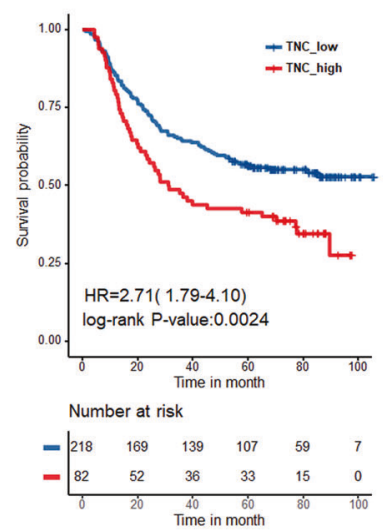

F

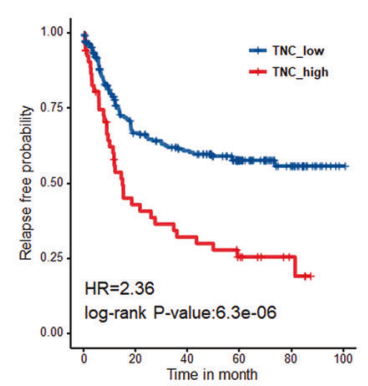

$$
\text { Number at risk }
$$$$
=\begin{array}{cccccc}
247 & 130 & 108 & 59 & 25 & 1 \\
53 & 20 & 15 & 10 & 4 & 0 \\
\hline 0 & 20 & 40 & 60 & 80 & 100
\end{array}
$$

G

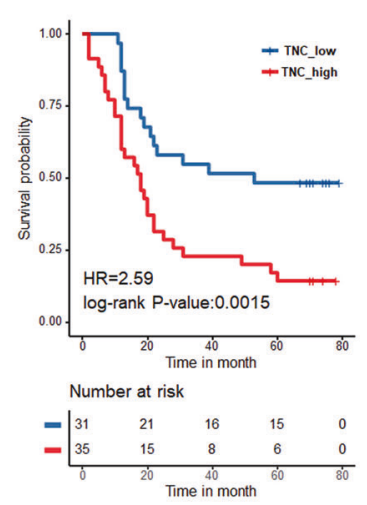

Fig. 1 TNC was upregulated in gastric cancer tissues and correlated with poor prognosis in gastric cancer patients. A A representative IHC staining of normal and tumor tissue (magnification, $\times 100$ and $\times 200$ ). B TNC expression was significantly increased in gastric cancer tissues compared with adjust tissue. C Western blot analysis of TNC expression in gastric tissues and adjust tissue in eight patients. D IHC analysis of TNC, PAS and CD31 in gastric tissues; overall survival (E) and relapse-free survival (F) in patients with different TNC expression. Overall survival (G) of our single-center patients with different TNC expression. Results were shown as mean \pm SD of three independent experiments; each experiment was performed in triplicate. ${ }^{* * *} P<0.0001$. 
Table 2. Clinicopathological information of patients with gastric cancer.

\begin{tabular}{|c|c|c|c|}
\hline \multirow[t]{2}{*}{ Variable } & \multicolumn{2}{|l|}{ TNC expression } & \multirow[t]{2}{*}{$P$ value } \\
\hline & Strong $(n=35)$ & Weak $(n=31)$ & \\
\hline Age at diagnosis (years) & $63.19 \pm 8.861$ & $61.19 \pm 8.199$ & 0.242 \\
\hline Gender & & & 0.092 \\
\hline Male & 12 & 5 & \\
\hline Female & 23 & 26 & \\
\hline Lauren classification & & & 0.650 \\
\hline Intestinal & 13 & 15 & \\
\hline Diffuse & 10 & 7 & \\
\hline Mixed & 12 & 9 & \\
\hline Tumor size $(\mathrm{cm})$ & & & $0.008^{*}$ \\
\hline$<5$ & 10 & 19 & \\
\hline$\geq 5$ & 25 & 12 & \\
\hline Tumor differentiation & & & 0.308 \\
\hline Low & 24 & 18 & \\
\hline Medium & 10 & 10 & \\
\hline High & 1 & 3 & \\
\hline AJCC stage & & & $0.003^{*}$ \\
\hline 1 & 1 & 8 & \\
\hline ॥ & 7 & 9 & \\
\hline III & 27 & 14 & \\
\hline T stage & & & $0.003^{*}$ \\
\hline $\mathrm{T} 1$ & 1 & 4 & \\
\hline $\mathrm{T} 2$ & 0 & 4 & \\
\hline T3 & 23 & 20 & \\
\hline T4 & 11 & 3 & \\
\hline $\mathrm{N}$ stage & & & $0.011^{*}$ \\
\hline No & 6 & 13 & \\
\hline N1 & 5 & 7 & \\
\hline N2 & 5 & 2 & \\
\hline N3 & 19 & 9 & \\
\hline Nervous invasion & & & 0.250 \\
\hline Positive & 29 & 22 & \\
\hline Negative & 6 & 9 & \\
\hline Venous invasion & & & 0.082 \\
\hline Positive & 30 & 21 & \\
\hline Negative & 5 & 10 & \\
\hline
\end{tabular}

AJCC American Joint Committee on Cancer, 8th edition. ${ }^{*} P<0.05$.

mediated TNC knockdown. The transfection efficiency was confirmed by western blot and quantitative real-time PCR (Fig. $2 B, C)$. The $2 \#$ siRNA was found to deliver the most effective knockdown and was used to construct lentivirus. The knockdown efficiency of lentivirus was examined by western blot (Fig. 2D). Then, we used bioinformatic analysis to explore the potential function of TNC and found that the function of TNC was strongly related to angiogenesis (Fig. 2E). Thus we boldly hypothesize that TNC is closely related to vasculogenic mimicry. Following this, we found that VM was significantly reduced in these cells after transfection with TNC shRNA (Fig. 2F).

Knockdown of TNC inhibited the proliferation, migration and invasion of gastric cancer cells

Since VM formation is closely related to the proliferation, invasion and migration of tumor cells [26], we evaluated the detailed role of TNC in gastric cancer cells. CCK-8 assays showed that the viability of AGS, BGC-823 and Hs746T cells was significantly decreased in the shTNC group compared to the control group (Fig. 3A). Consistent with this, the colony formation assay (Fig. 3B) and EdU thymidine analog incorporation assay (Fig. 3C) also indicated that knockdown of TNC inhibited the proliferation of AGS, BGC-823 and Hs746T cells compared with the control group. What's more, knockdown of TNC caused cell cycle arrest in the G2/ $M$ phase, preventing cell cycle progression from the $S$ phase into the $M$ phase in AGS, BGC-823 and Hs746T cells (Fig. 3D). Flow cytometry also showed that TNC knockdown increased Annexin V-positive cells in AGS, BGC-823 and Hs746T cells indicating that TNC knockdown induced apoptosis in gastric cancer cells (Fig. 3E). The migration of AGS, BGC-823 and Hs746T cells was evaluated through wound-healing (Fig. $3 \mathrm{~F}$ ) and transwell migration assays (Fig. 3G), indicating that TNC knockdown inhibited the migration rates of gastric cancer cells. Transwell assays (Fig. $3 \mathrm{H}$ ) and pseudopodia formation assay (Fig. 3l) also revealed a significant reduction in invasion upon TNC knockdown.

\section{Knockdown of TNC inhibited ERK phosphorylation and EMT process}

VM formation involves numerous factors including ERK, MMP2/ 9 and EMT process $[9,26-28]$. Thus, we used Gene Set Enrichment Analysis to analyze the correlation between TNC expression in gastric cancer and activation of the MAPK pathway and found a significant positive association between TNC expression and activation of the ERK pathway (Fig. 4A). Bioinformatic analysis also showed that TNC expression was in accordance with EMT process (Fig. 4B). Subsequently, we examined the mRNA (Fig. 4C) and protein (Fig. 4D) levels of phospho-ERK, MMP2/9 and markers of EMT and found that phpspho-ERK, MMP2/9 were significantly decreased and gastric cancer cells was induced to proceed mesenchymal-to-epithelial transition process upon TNC knockdown.

Knockdown of TNC inhibited VM through ERK-mediated EMT To determine whether ERK is associated with MMP2/9, EMT process and VM formation, PD98059, an ERK1/2 inhibitor, was used to conduct rescue experiment. As expected, VM formation of AGS, BGC-823 and Hs746T cells was reduced in a dosedependent manner after treatment with $0,5,10$ and $20 \mu \mathrm{M}$ PD98059 for $24 \mathrm{~h}$ (Fig. 5A). What's more, phosphor-ERK, MMP2/9, $\mathrm{N}$-cadherin, Vimentin, Snail, Slug and Twist1 were downregulated and E-cadherin was upregulated in gastric cancer cells upon exposure to PD98059 at 0, 5, 10, $20 \mu \mathrm{M}$ for $24 \mathrm{~h}$ (Fig. 5B, C). $A$ rescue assay revealed that $\mathrm{VM}$ formation of $\mathrm{AGS}, \mathrm{BGC}-823$ and Hs746T cells transfected with shTNC was increased upon treatment with LM22B-10, an activator of ERK (Fig. 5D). The same changes were also observed in protein levels of phosphorERK, MMP2/9 and EMT markers (Fig. 5E, F), further supporting this conclusion.

\section{Knockdown of TNC inhibits tumor growth and peritoneal dissemination in vivo}

To determine the effects of TNC in vivo, xenograft and peritoneal dissemination model were established with AGS cells infected with lentivirus expressing shRNA-NC or shRNA-TNC. For xenograft model, 21 days after subcutaneous injection, mice were sacrificed, and the weight and volume of tumors were measured. The weight and volume of tumors in TNC knockdown group were decreased compared to the control group (Fig. 6A-C). Furthermore, TNC, VM (CD31-/PAS+), MMP2 and MMP9 in AGS xenograft tissues were significantly decreased in shTNC groups compared with that in the NC group (Fig. 6D). For peritoneal dissemination model, 14 days after peritoneal cavities injection, mice were sacrificed. The results showed that 

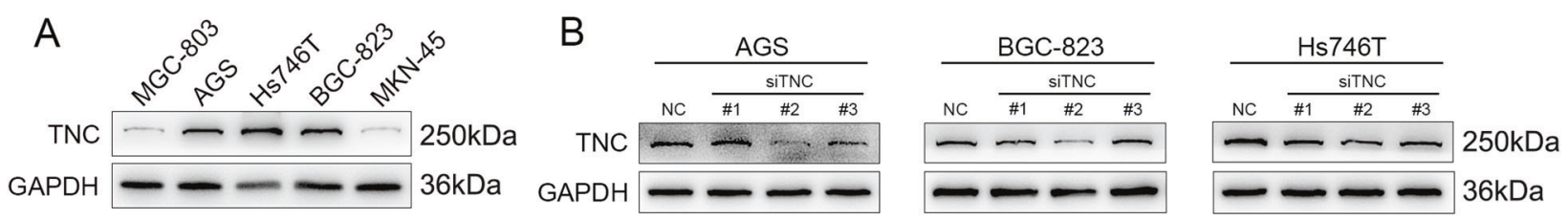

C
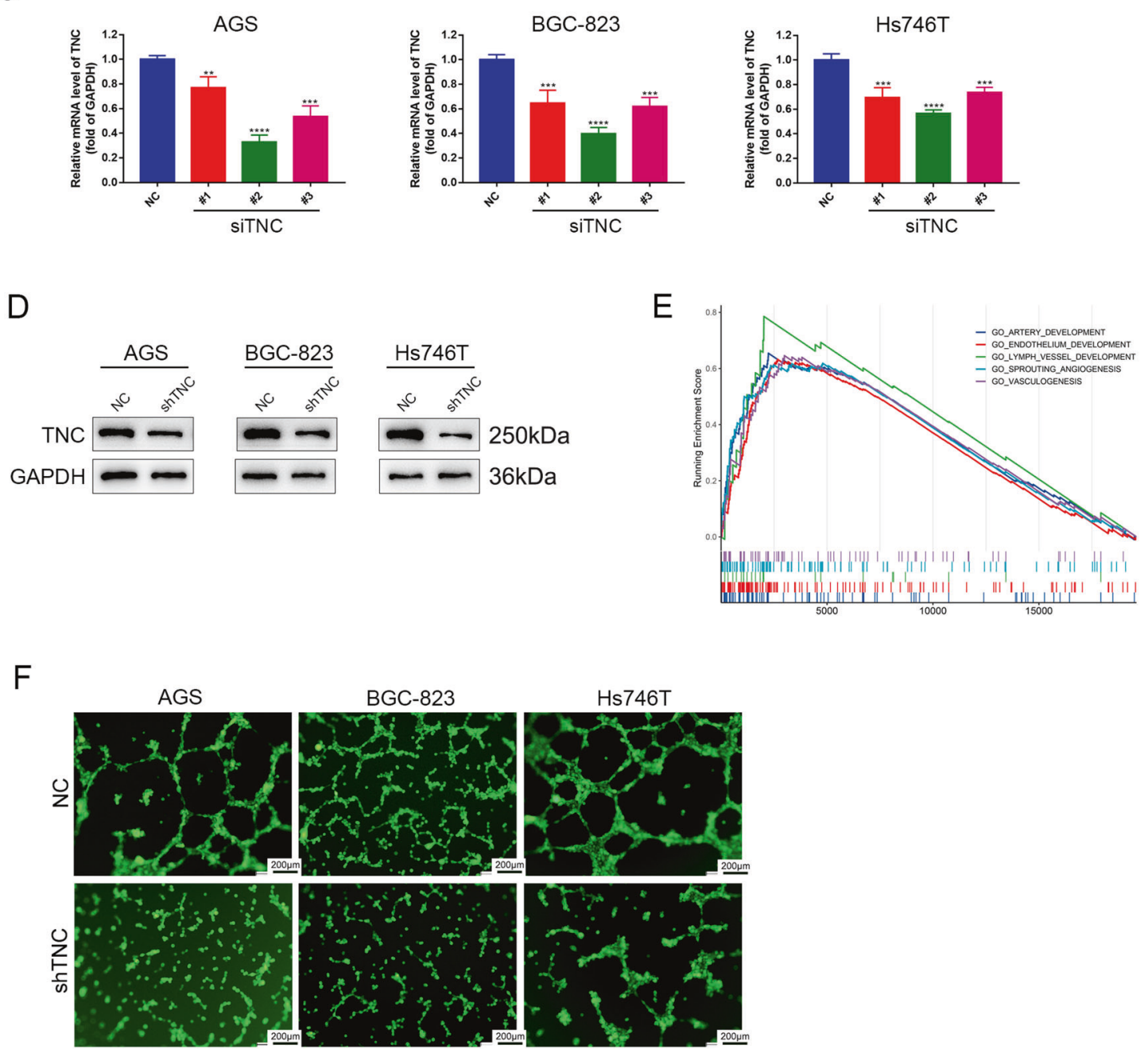

Fig. 2 Construction of cell lines and TNC knockdown attenuated VM formation in vitro. A Western blot of TNC protein and gene expression in four gastric cancer cell lines; GAPDH was used as a loading control; protein (B) and gene (C) expression levels of TNC in AGS, BGC-823 and Hs746T cells transfected with siRNA targeting TNC. D Western blot analysis of knockdown efficiency of shTNC. E Bioinformation analysis of TNC functions. F VM was significantly reduced in AGS, BGC-823 and Hs746T cells after transfection with TNC shRNA. Results were shown as mean \pm SD of three independent experiments; each experiment was performed in triplicate. ${ }^{* *} P<0.01 ;{ }^{* *} P<0.001 ;{ }^{* * * *} P<0.0001$.

knockdown of TNC reduced the mesenteric metastatic nodules in the intestinal wall of nude mice (Fig. $6 \mathrm{E}, \mathrm{F}$ ).

\section{DISCUSSION}

Gastric cancer is one of the most common malignant tumors in the world [2] and recurrence and metastasis are the causes of the death in patients with gastric cancer. Once patients develop resistance to chemotherapeutics, anti-angiogenesis therapy becomes an important method to treat gastric cancer [29]. However, the effect of anti-angiogenesis therapy is not satisfactory and VM and metastasis continue to increase thereafter [30]. It has been reported that VM plays an important role in regulating the progression and metastasis in gastric cancer $[11,16]$. However, the mechanism underlying VM formation in gastric cancer is unclear and it is urgent to seek therapies targeting VM formation.

TNC is an extracellular matrix protein and was reported to induce VM in glioma [26] and melanoma [31]. Recent studies demonstrated that TNC was strongly expressed and indicated poor prognosis in gastric cancer $[16,20]$. The present results confirmed that TNC was upregulated in gastric cancer tissues and had a positive correlation with VN, and patients with higher TNC levels had significantly shorter OS and RFS compared to those with low TNC expression, consistent with our single-center data. Then, we evaluated VM formation in gastric cancer cell lines and 
A

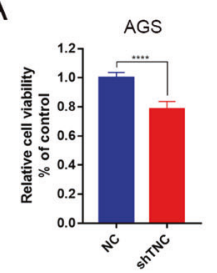

B
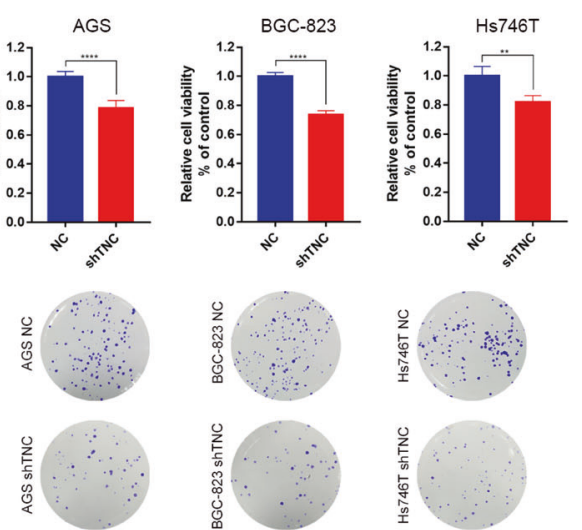

D
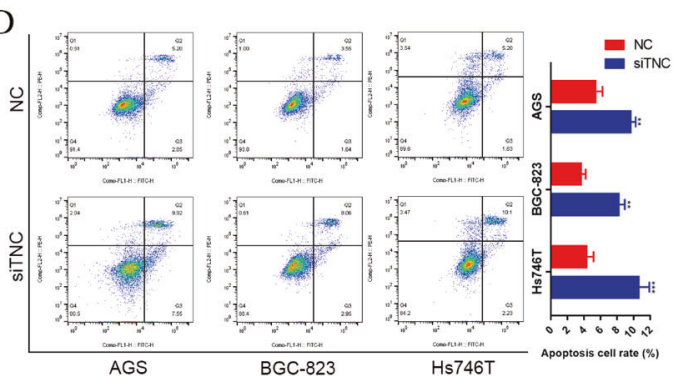

C
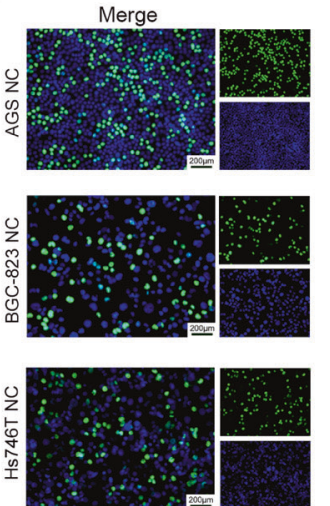

E
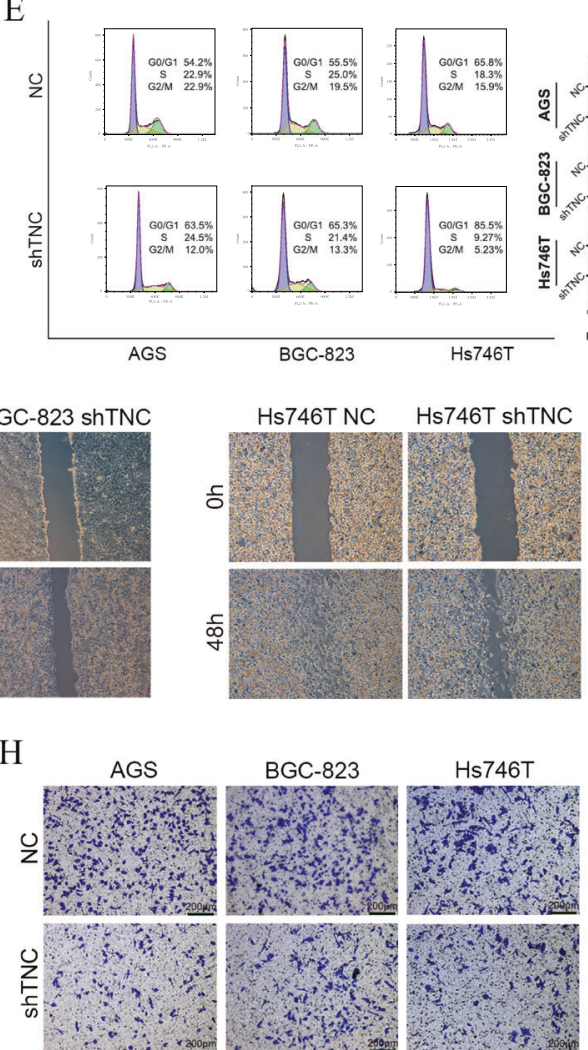

$\mathrm{G}$
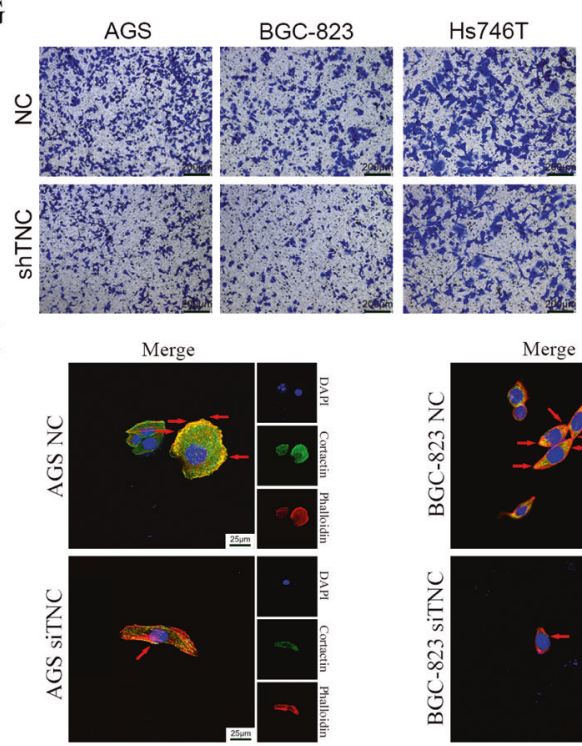

$\mathrm{H}$
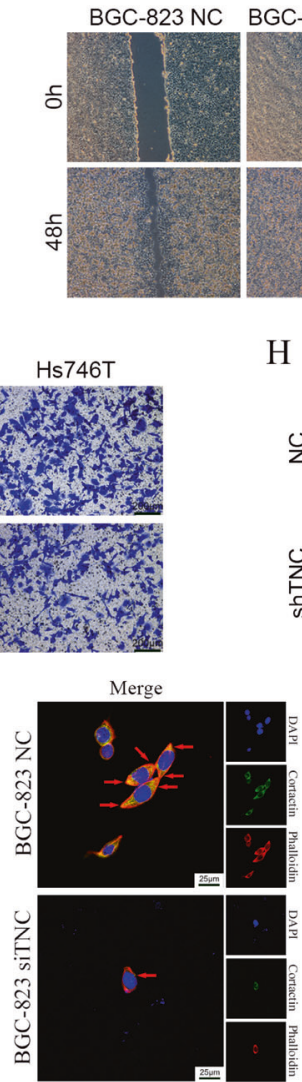

Fig. 3 TNC knockdown inhibited the proliferation, migration and invasion of gastric cancer cells. CCK-8 (A), colony formation assay (B) and EdU assay (C) showed that TNC knockdown inhibited the proliferation of gastric cancer cells; flow cytometry showed that TNC induced cell cycle arrest $(\mathbf{D})$ and apoptosis $(\mathbf{E})$ in gastric cancer cells; cell migration was detected by wound-healing assay $(\mathbf{F})$ and transwell assay $(\mathbf{G})$; cell invasion was detected by transwell assay $(\mathbf{H})$ and pseudopodia formation assay $(\mathbf{I})$. Results were shown as mean \pm SD of three independent experiments; each experiment was performed in triplicate. ${ }^{* *} P<0.01$; ${ }^{* * * *} P<0.0001$. 
A

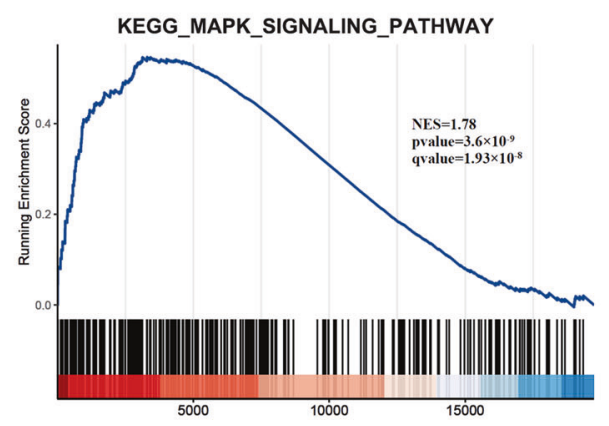

C
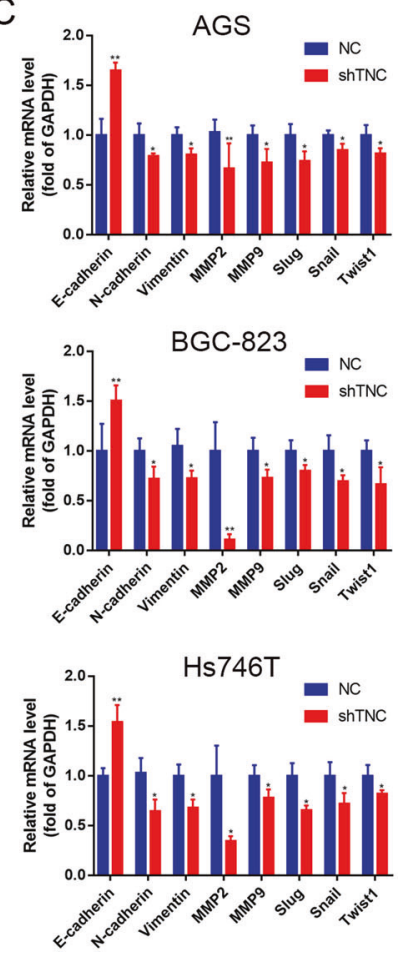

B

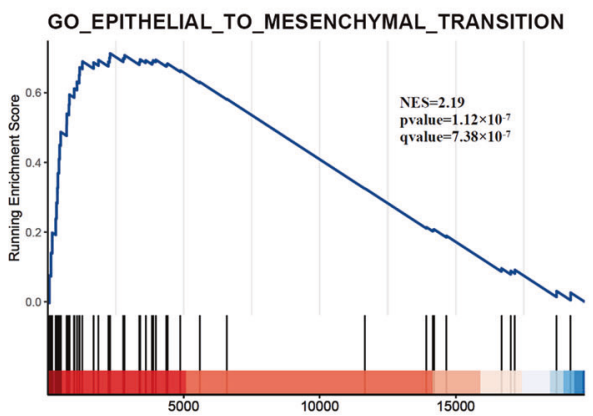

$\mathrm{D}$

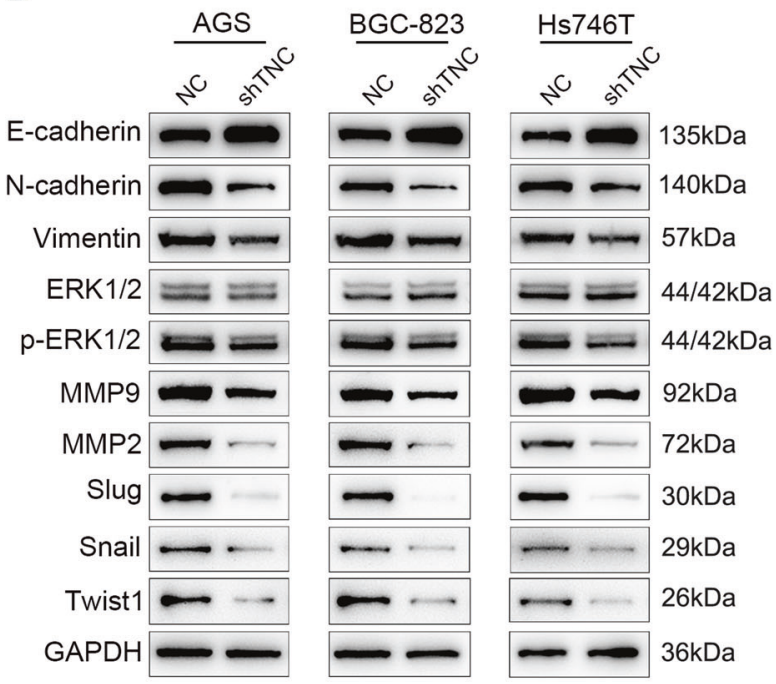

Fig. 4 TNC correlated with ERK pathway and EMT process. A Gene Set Enrichment Analysis evaluating TNC expression and the MAPK_SIGNALING pathway in gastric cancer. B Bioinformatic analysis showed that TNC expression was in accordance with EMT process; qPCR (C) and western blot analysis (D) of ERK and EMT markers expression in AGS, BGC-823 and Hs746T cells transfected with TNC siRNA.

found that TNC knockdown inhibited the VM formation ability in vitro and in vivo. Furthermore, knockdown of TNC suppressed the proliferation of gastric cancer cell lines and subcutaneous tumor and decreased the number of cells in the G0/G1 phase, indicating that TNC knockdown inhibited the proliferation of gastric cancer cell lines by inducing the cell cycle arrest in the G0/ G1 phase. Concurrently, knockdown of TNC also inhibited the migration and invasion of gastric cancer cell in vitro and inhibited peritoneal metastasis in vivo.

To further investigate the mechanism underlying TNC and VM formation, we used bioinformatic analysis and found that TNC was positively associated with MAPK pathway and EMT process. As is known, EMT plays an important role in VM formation $[9,32]$ and MMP2/9 are important downstream effectors of ERK and contribute to VM formation [33]. Then, we investigated the correlation of TNC and VM with EMT markers in gastric cancer cells and found that TNC was positively correlated with N-cadherin, Vimentin, MMP2/9 and negatively related to E-cadherin, consistent with previous research $[34,35]$. These results preliminarily indicated that TNC may promote VM formation by inducing ERK-triggered EMT. Then, we used PD98059 to inhibit ERK phosphorylation and found that VM and EMT process were decreased in a dose-dependent manner. What's more, VM formation and EMT process of TNC knockdown cells were reversed by LM22B-10, further proving the hypothesis.

In conclusion, our results demonstrated that TNC knockdown blocked the EMT process by suppressing the ERK pathway, leading to the inhibition of VM formation. The combined targeting of TNC and ERK pathway may provide a potential antitumor therapy for inhibiting VM formation and decrease antiangiogenic therapeutic resistance.

\section{DATA AVAILABILITY}

The data supporting our conclusion were obtained from the TCGA database (https:// cancergenome.nih.gov). All the data supporting the conclusions were included in the main paper. 
A

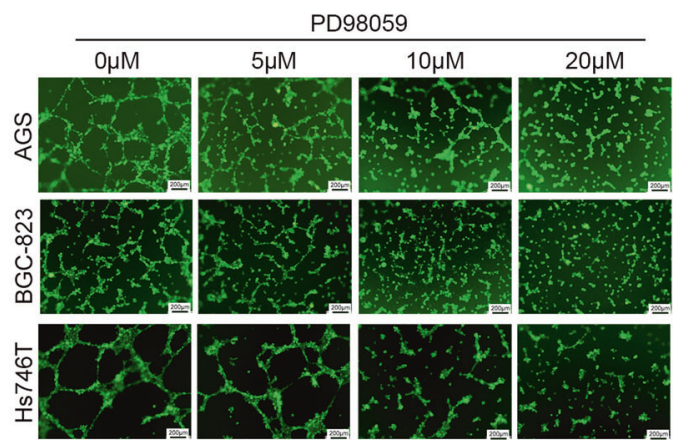

$\mathrm{C}$

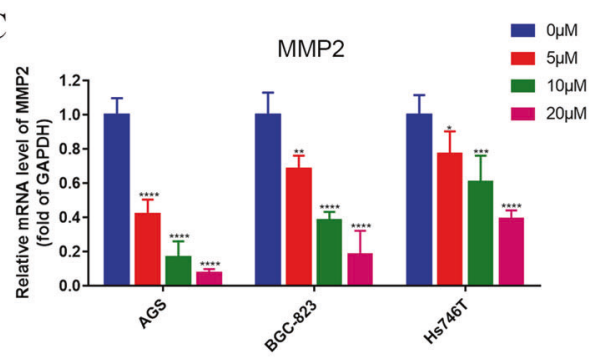

D

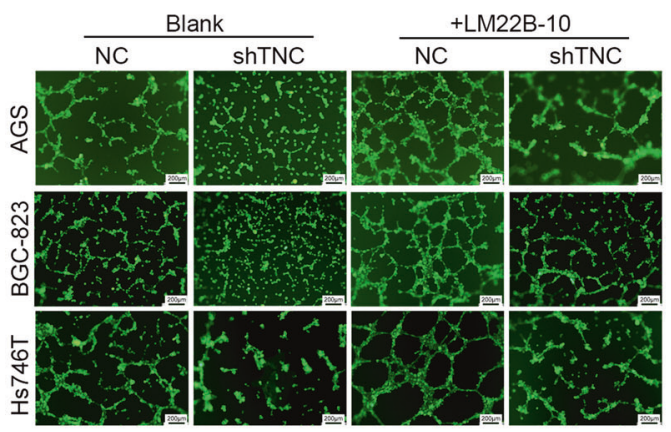

B
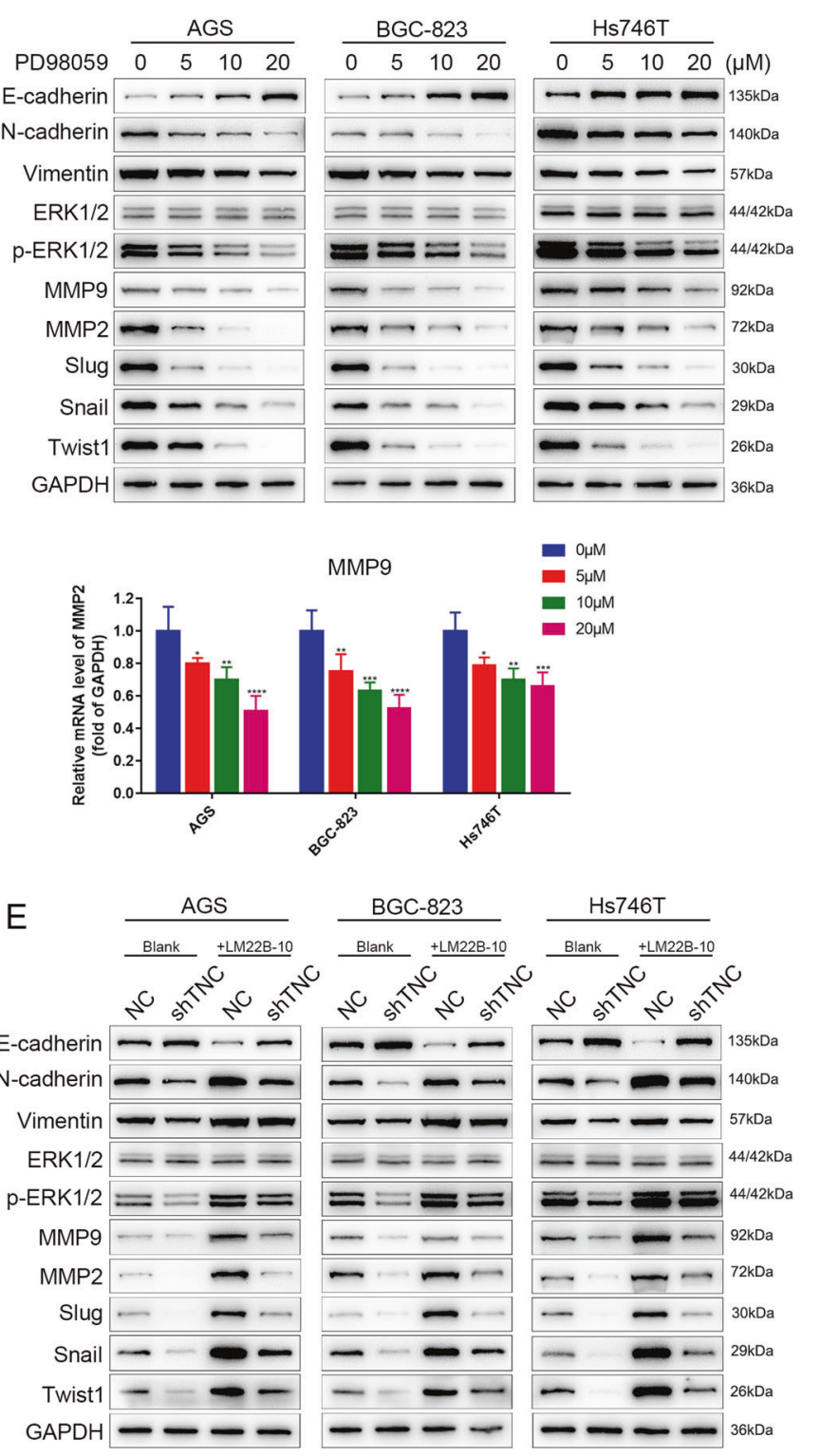
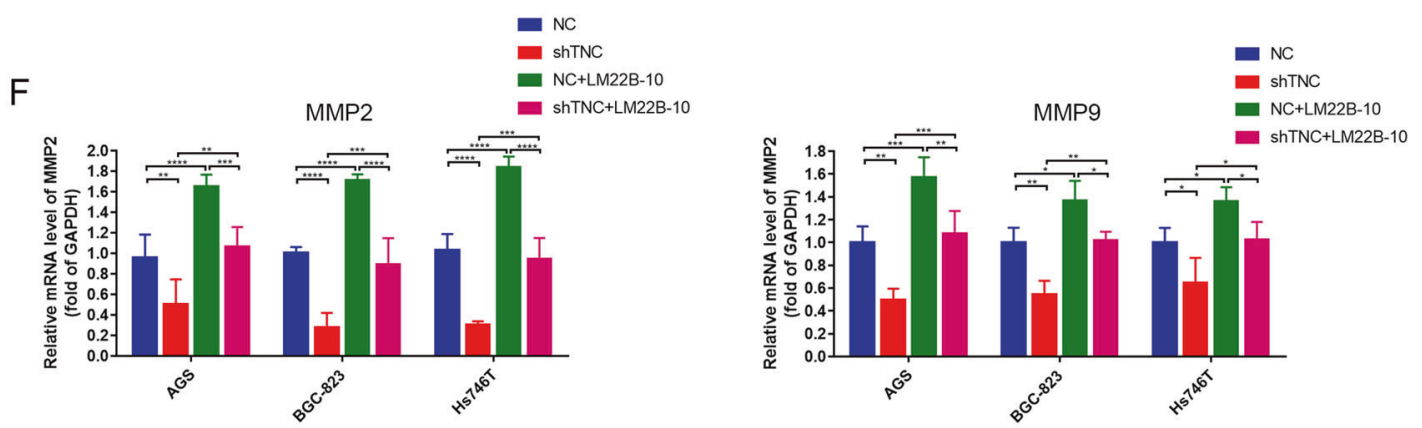

Fig. 5 Knockdown of TNC inhibited VM through ERK-mediated EMT. A VM formation was inhibited in AGS, BGC-823 and Hs746T cells after exposure to the ERK inhibitor PD98059. B Western blot of ERK and EMT markers expression in AGS, BGC-823 and Hs746T cells after exposure to PD98059. C qPCR analysis of MMP2/9 in AGS, BGC-823 and Hs746T cells after exposure to PD98059. D VM formation of AGS, BGC-823 and Hs746T cells transfected with shTNC were increased upon treatment with the ERK activator LM22B-10. E Western blot of ERK and EMT markers expression in AGS, Hs746T and BGC-823 cells transfected with shTNC and treated with LM22B-10. F qPCR analysis of MMP2/9 in AGS, BGC-823 and Hs746T cells transfected with siTNC and treated with LM22B-10. Results were shown as mean \pm SD of three independent experiments; each experiment was performed in triplicate. ${ }^{*} P<0.05 ;{ }^{* *} P<0.01 ;{ }^{* * *} P<0.001 ;{ }^{* * *} P<0.0001$. 
A

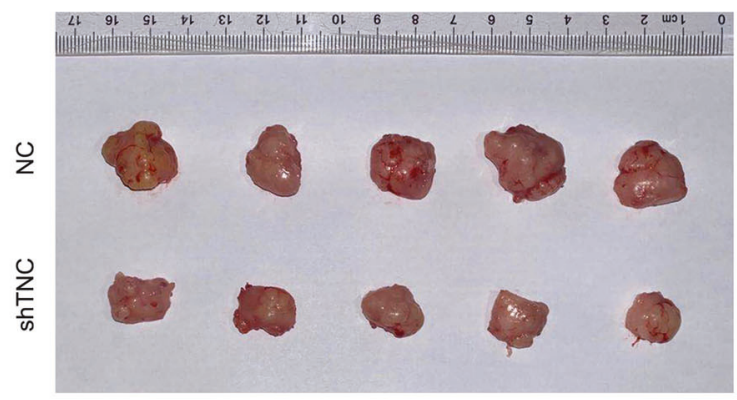

B

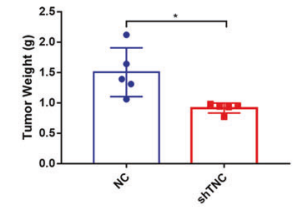

C

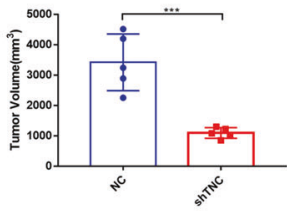

D
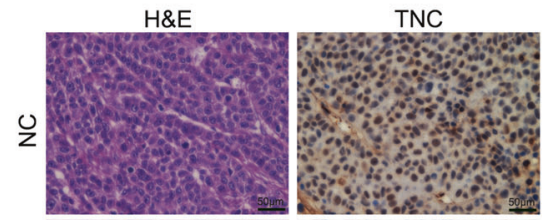

CD31/PAS
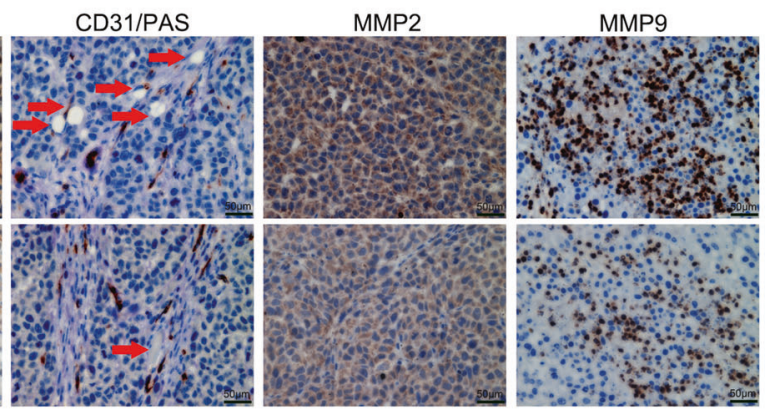

E
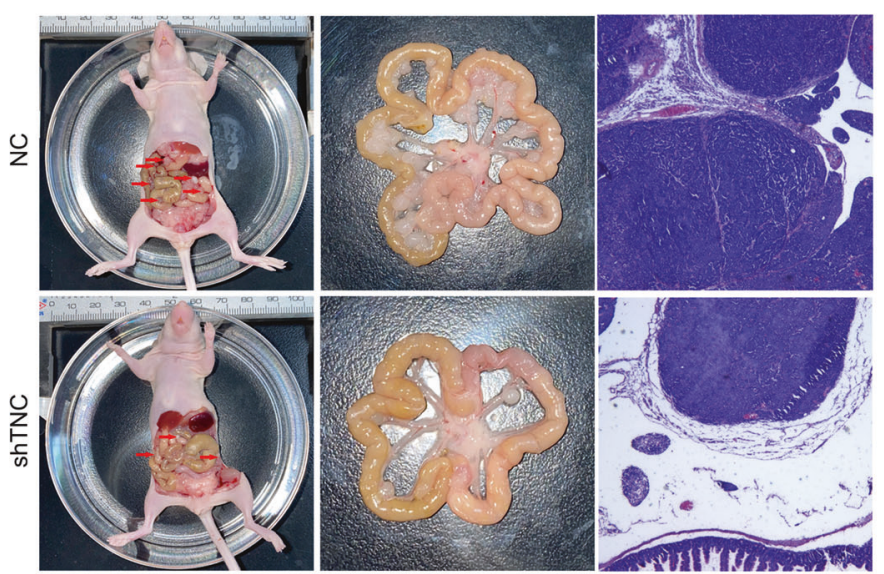

F

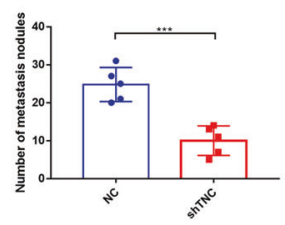

Fig. 6 TNC knockdown inhibited tumor growth and peritoneal dissemination in vivo. A Image of tumors of mice in the NC and shTNC groups. B Volume of tumor of mice in the NC and shTNC groups. C Weight of tumor of mice in the NC and shTNC groups. D H\&E, TNC, VM (CD31-/PAS+), MMP2 and MMP9 staining in NC and shTNC xenograft tissues. E Representative intestines in nude mice. F The quantification of metastases in nude mice was counted. Results were shown as mean \pm SD of three independent experiments; each experiment was performed in triplicate. ${ }^{*} P<0.05$; ${ }^{* * *} P<0.001$.

\section{MATERIALS AVAILABILITY}

All the materials supporting the conclusions were included in the main paper.

\section{REFERENCES}

1. Chen W, Zheng R, Baade PD, Zhang S, Zeng H, Bray F, et al. Cancer statistics in China, 2015. CA Cancer J Clin. 2016;66:115-32.

2. Bray F, Ferlay J, Soerjomataram I, Siegel RL, Torre LA, Jemal A. Global cancer statistics 2018: GLOBOCAN estimates of incidence and mortality worldwide for 36 cancers in 185 countries. CA Cancer J Clin. 2018;68:394-424.

3. Feng RM, Zong YN, Cao SM, Xu RH. Current cancer situation in China: good or bad news from the 2018 Global Cancer Statistics? Cancer Commun. 2019;39:22.

4. Thrift AP, El-Serag HB. Burden of gastric cancer. Clin Gastroenterol Hepatol. 2020;18:534-42.

5. Correa P. Gastric cancer: overview. Gastroenterol Clin North Am. 2013;42:211-7.
6. Dong $X$, Hong $Y$, Sun $H$, Chen $C$, Zhao $X$, Sun B. NDRG1 suppresses vasculogenic mimicry and tumor aggressiveness in gastric carcinoma. Oncol Lett. 2019;18:3003-16.

7. McLemore MR. The role of the data safety monitoring board: why was the Avastin phase III clinical trial stopped? Clin J Oncol Nurs. 2006;10:153-4.

8. Maniotis AJ, Folberg R, Hess A, Seftor EA, Gardner LM, Pe'er J, et al. Vascular channel formation by human melanoma cells in vivo and in vitro: vasculogenic mimicry. Am J Pathol. 1999;155:739-52.

9. Liu Q, Qiao L, Liang N, Xie J, Zhang J, Deng G, et al. The relationship between vasculogenic mimicry and epithelial-mesenchymal transitions. J Cell Mol Med. 2016;20:1761-9.

10. Folberg R, Maniotis AJ. Vasculogenic mimicry. APMIS. 2004;112:508-25.

11. Qu B, Guo L, Ma J, Lv Y. Antiangiogenesis therapy might have the unintended effect of promoting tumor metastasis by increasing an alternative circulatory system. Med Hypotheses. 2010;74:360-1. 
12. Liu T, Sun B, Zhao X, Li Y, Gu Q, Dong X, et al. OCT4 expression and vasculogenic mimicry formation positively correlate with poor prognosis in human breast cancer. Int J Mol Sci. 2014;15:19634-49.

13. Zhao N, Sun BC, Zhao XL, Wang Y, Meng J, Che N, et al. Role of $\mathrm{BCl}-2$ and its associated miRNAs in vasculogenic mimicry of hepatocellular carcinoma. Int $J$ Clin Exp Pathol. 2015;8:15759-68.

14. Schnegg $\mathrm{Cl}$, Yang MH, Ghosh SK, Hsu MY. Induction of vasculogenic mimicry overrides VEGF-A silencing and enriches stem-like cancer cells in melanoma. Cancer Res. 2015;75:1682-90.

15. Li W, Zong S, Shi Q, Li H, Xu J, Hou F. Hypoxia-induced vasculogenic mimicry formation in human colorectal cancer cells: involvement of HIF-1a, Claudin-4, and E-cadherin and Vimentin. Sci Rep. 2016;6:37534.

16. Guo Q, Yuan Y, Jin Z, Xu T, Gao Y, Wei H, et al. Association between tumor vasculogenic mimicry and the poor prognosis of gastric cancer in China: an updated systematic review and meta-analysis. Biomed Res Int. 2016;2016:2408645.

17. Zhou L, Yu L, Feng ZZ, Gong XM, Cheng ZN, Yao N, et al. Aberrant expression of markers of cancer stem cells in gastric adenocarcinoma and their relationship to vasculogenic mimicry. Asian Pac J Cancer Prev. 2015;16:4177-83.

18. Midwood KS, Chiquet M, Tucker RP, Orend G. Tenascin-C at a glance. J Cell Sci. 2016;129:4321-7.

19. Xia S, Lal B, Tung B, Wang S, Goodwin CR, Laterra J. Tumor microenvironment tenascin-C promotes glioblastoma invasion and negatively regulates tumor proliferation. Neuro Oncol. 2016;18:507-17.

20. Qi W, Yang Z, Li H, Cui Y, Xuan Y. The role of Tenascin-C and Twist1 in gastric cancer: cancer progression and prognosis. APMIS. 2019;127:64-71.

21. Hamza O, Kiss A, Kramer AM, Trojanek S, Abraham D, Acar E, et al. Tenascin C promotes valvular remodeling in two large animal models of ischemic mitral regurgitation. Basic Res Cardiol. 2020;115:76.

22. Han C, Sun B, Zhao X, Zhang Y, Gu Q, Liu F, et al. Phosphorylation of STAT3 promotes vasculogenic mimicry by inducing epithelial-to-mesenchymal transition in colorectal cancer. Technol Cancer Res Treat. 2017;16:1209-19.

23. Sun $T$, Sun $B C$, Zhao $X L$, Zhao N, Dong $X Y, C$ he $N$, et al. Promotion of tumor cell metastasis and vasculogenic mimicry by way of transcription coactivation by $\mathrm{Bcl}-2$ and Twist1: a study of hepatocellular carcinoma. Hepatology. 2011;54:1690-706.

24. You X, Wu J, Wang Y, Liu Q, Cheng Z, Zhao X, et al. Galectin-1 promotes vasculogenic mimicry in gastric adenocarcinoma via the Hedgehog/GLI signaling pathway. Aging. 2020;12:21837-53.

25. You X, Liu Q, Wu J, Wang Y, Dai J, Chen D, et al. Galectin-1 promotes vasculogenic mimicry in gastric cancer by upregulating EMT signaling. J Cancer. 2019;10:6286-97.

26. Cai HP, Wang J, Xi SY, Ni XR, Chen YS, Yu YJ, et al. Tenascin-cmediated vasculogenic mimicry formation via regulation of MMP2/MMP9 in glioma. Cell Death Dis. 2019;10:879.

27. Li W, Zhou Y. LRIG1 acts as a critical regulator of melanoma cell invasion, migration, and vasculogenic mimicry upon hypoxia by regulating EGFR/ERKtriggered epithelial-mesenchymal transition. Biosci Rep. 2019;39:BSR20181165.

28. Meng J, Chen S, Lei YY, Han JX, Zhong WL, Wang XR, et al. Hsp90beta promotes aggressive vasculogenic mimicry via epithelial-mesenchymal transition in hepatocellular carcinoma. Oncogene. 2019;38:228-43.

29. Chen LT, Oh DY, Ryu MH, Yeh KH, Yeo W, Carlesi R, et al. Anti-angiogenic therapy in patients with advanced gastric and gastroesophageal junction cancer: a systematic review. Cancer Res Treat. 2017;49:851-68.

30. Xu Y, Li Q, Li XY, Yang OY, Xu WW, Liu GL. Short-term anti-vascular endothelial growth factor treatment elicits vasculogenic mimicry formation of tumors to accelerate metastasis. J Exp Clin Cancer Res. 2012;31:16.

31. Verrando P, Capovilla M, Rahmani R. Trans-nonachlor decreases miR-141-3p levels in human melanocytes in vitro promoting melanoma cell characteristics and shows a multigenerational impact on miR-8 levels in Drosophila. Toxicology. 2016;368-369:129-41.

32. Fan $\mathrm{YL}$, Zheng $\mathrm{M}$, Tang $\mathrm{YL}$, Liang $\mathrm{XH}$. A new perspective of vasculogenic mimicry: EMT and cancer stem cells (Review). Oncol Lett. 2013;6:1174-80.

33. Zhang JG, Zhang DD, Liu $Y$, Hu JN, Zhang $X$, Li L, et al. RhoC/ROCK2 promotes vasculogenic mimicry formation primarily through ERK/MMPs in hepatocellular carcinoma. Biochim Biophys Acta Mol Basis Dis. 2019;1865:1113-25.

34. Xu Y, Li Z, Jiang P, Wu G, Chen K, Zhang X, et al. The co-expression of MMP-9 and Tenascin- $C$ is significantly associated with the progression and prognosis of pancreatic cancer. Diagn Pathol. 2015;10:211.
35. Yang Z, Zhang C, Qi W, Cui C, Cui Y, Xuan Y. Tenascin-C as a prognostic determinant of colorectal cancer through induction of epithelial-to-mesenchymal transition and proliferation. Exp Mol Pathol. 2018;105:216-22.

\section{ACKNOWLEDGEMENTS}

This work was supported by the National Ministry of Science and Technology Project (No. 2016YFC0104105), the Key Research Plan and Social Development Project of Jiangsu Province, China (No. BE2016603) and the Distinguished Young Scholar Project of Medical Science and Technology Development Foundation of Nanjing Department of Health (No. JQX19001)

\section{AUTHOR CONTRIBUTIONS}

XK and EX analyzed and interpreted data, and drafted the manuscript. XW and LQ made acquisition of data, and performed statistical analysis. ZY, HY, CW, CR, YW and $\mathrm{XL}$ participated in studies selection and data extraction and provided statistical expertise. XX, WG and TQ conceived of the study, participated in its design, analyzed and interpreted the data. All authors read and approved the final manuscript.

\section{COMPETING INTERESTS}

The authors declare no competing interests.

\section{ETHICS APPROVAL AND CONSENT TO PARTICIPATE}

The use of the clinical samples was approved by the Hospital Ethics Committee of Nanjing Drum Tower Hospital (The Affiliated Hospital of Nanjing University School), and carried out in accordance with the principles of the Declaration of Helsinki and informed consent was obtained from all subjects. All animal experiments are conducted in accordance with the principles and procedures approved by the Hospital Ethics Committee of Nanjing Drum Tower Hospital (The Affiliated Hospital of Nanjing University School).

\section{CONSENT FOR PUBLICATION}

All authors have agreed to publish this manuscript.

\section{ADDITIONAL INFORMATION}

Correspondence and requests for materials should be addressed to Xuefeng Xia, Wenxian Guan or Tong Qiao.

Reprints and permission information is available at http://www.nature.com/ reprints

Publisher's note Springer Nature remains neutral with regard to jurisdictional claims in published maps and institutional affiliations.

Open Access This article is licensed under a Creative Commons Attribution 4.0 International License, which permits use, sharing, adaptation, distribution and reproduction in any medium or format, as long as you give appropriate credit to the original author(s) and the source, provide a link to the Creative Commons license, and indicate if changes were made. The images or other third party material in this article are included in the article's Creative Commons license, unless indicated otherwise in a credit line to the material. If material is not included in the article's Creative Commons license and your intended use is not permitted by statutory regulation or exceeds the permitted use, you will need to obtain permission directly from the copyright holder. To view a copy of this license, visit http://creativecommons. org/licenses/by/4.0/.

(c) The Author(s) 2021, corrected publication 2021 